\title{
A highly contrasting scanning helium microscope
}

\author{
A. Fahy, M. Barr, J. Martens, and P. C. Dastoor ${ }^{\text {a) }}$ \\ Centre for Organic Electronics, University of Newcastle, Callaghan, NSW 2308, Australia
}

(Received 28 December 2014; accepted 23 January 2015; published online 12 February 2015)

\begin{abstract}
We present a scanning helium microscope equipped to make use of the unique contrast mechanisms, surface sensitivity, and zero damage imaging the technique affords. The new design delivers an order of magnitude increase in the available helium signal, yielding a higher contrast and signalto-noise ratio. These improvements allow the microscope to produce high quality, intuitive images of samples using topological contrast, while setting the stage for investigations into further contrast mechanisms. (C) 2015 AIP Publishing LLC. [http://dx.doi.org/10.1063/1.4907539]
\end{abstract}

\section{INTRODUCTION}

An emerging technique capable of imaging without incurring the typical damage or degradation associated with more energetic techniques (such as electrons or ions) is the use of neutral atoms as the probe particle in a scanning helium microscope or SHeM. ${ }^{1}$ Using neutral helium, it is possible to design a surface sensitive microscopy capable of imaging delicate structures such as polymers, biological samples, and delicate adsorbate structures. ${ }^{1}$ The key to the lack of damage is (in part) the very low energy of the probe particle - typically 5-100 meV for a thermal energy helium atom. ${ }^{2}$ Based on the de Broglie wavelength of such atoms, the ultimate resolution of a scanning helium microscope is sub-Ångstrom, although technical limitations with regards to both detectors and neutral atom optics means that this limit is out of reach at present. However, research from several groups is making good progress on both fronts. ${ }^{2-8}$

Recent progress in SHeM development has involved an optical arrangement using a simple pinhole. ${ }^{9,10}$ The authors recently reported on a SHeM geometry (henceforth referred to as "SHeM I") designed to optimise the number of possible contrast mechanisms available to the technique through having the helium beam interact with the sample at $45^{\circ}$. By monitoring the specular position, we maximise the chances of detecting a difference between the specularly and diffusely scattered helium. The dependence of the helium scattering on the elemental composition of the surface as seen in helium atom scattering (HAS) means that such an interaction geometry should yield a form of material dependent contrast (in addition to topological and diffractive mechanisms), opening the potential for chemical fingerprinting in SHeM images. The pinhole size for this previous instrument was ultimately limited to 5 microns due to the available neutral helium signal at the detector - improving the helium signal corresponds to a direct improvement in resolution, or image collection time (through a decrease in the detector response time). More importantly, insufficient pumping in the differential pumping stage resulted in the creation of an effusive beam source during operation. While optimizing the nozzle-to-skimmer positioning could

\footnotetext{
a)E-mail: Paul.Dastoor@newcastle.edu.au
}

minimise its effects, this secondary beam would still act to degrade the produced image and complicate any contrast analysis.

Here, we present a new SHeM design which drastically improves the available signal by cutting the beam path length down while increasing the available pumping. The increase in count rate in the new instrument is quantified, both experimentally and through modelling, and the improved imaging capabilities demonstrated. Finally, the detrimental effects of the secondary effusive beam on the contrast and noise in a SHeM image are discussed.

\section{INSTRUMENT DESIGN}

As with the previous design, the new SHeM (henceforth referred to as "SHeM II") allows for the helium beam to interact with the sample surface at an angle of $45^{\circ}$, with collection at the same angle. A schematic is shown in Figure 1, outlining the path of the neutral helium from the beam source, through the differential pumping stage and pinhole optics, onto the sample, before finally registering a signal at the detector. The source is based on the design of Buckland et al., ${ }^{11}$ but with a simple modification to allow for effective chilling of the nozzle through liquid nitrogen. A 10 micron nozzle produces the supersonic free-jet expansion, with stagnation pressures up to 250 bar possible. A stagnation pressure of 200 bar at room temperature produces a centreline intensity of $(1.5 \pm 0.2) \times$ $10^{20}$ atoms/sec steradian ${ }^{-1}$, estimated by monitoring the source chamber pressure whilst the beam is in operation. The source chamber is pumped by an Edwards iXR-2206 turbomolecular pump capable of $2200 \mathrm{l} / \mathrm{s}$, giving a chamber base pressure of $5 \times 10^{-9}$ millibars when the beam is not in operation.

A sharp, cone-shaped skimmer (Beam Dynamics Inc. Model 2 with 100 micron aperture) samples the centreline of the expansion allowing a thin, intense beam of helium to pass through to the differential pumping stage. In order to reduce the beam path length whilst simultaneously improving the available pumping, the differential and sample chambers were built into the same box chamber, separated by an internal wall. The design allows a DN63CF Edwards EXT75DX turbo pump to be mounted directly to the differential chamber, dramatically improving the pump rate. The helium beam passes through the 


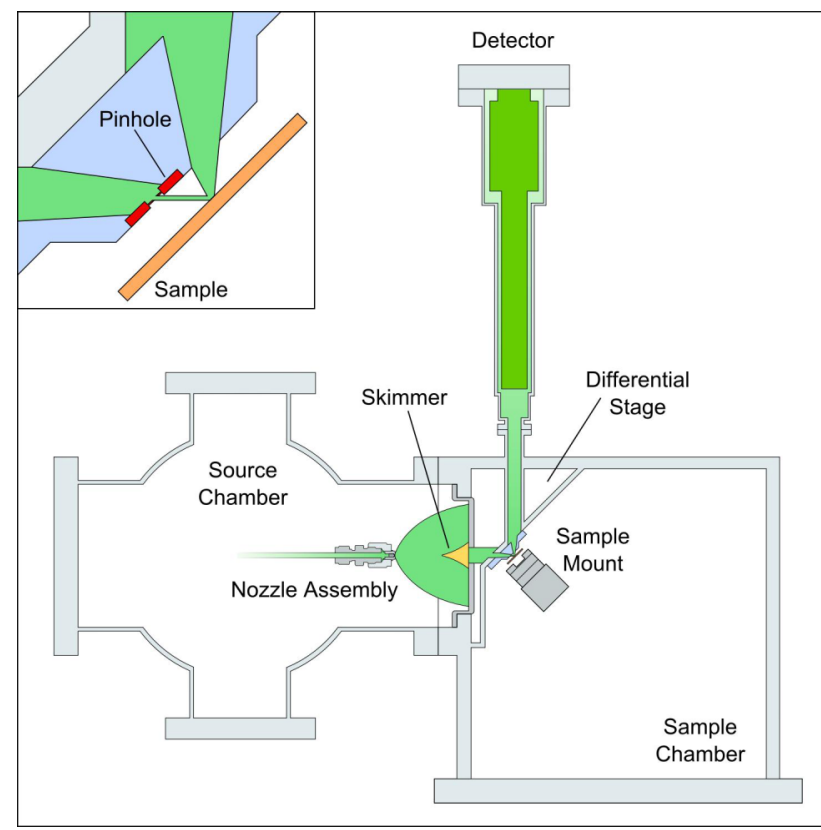

FIG. 1. Schematic diagram of the SHeM II system. Using a $10 \mu \mathrm{m}$ nozzle, a supersonic free-jet expansion of neutral helium is produced in the source chamber. A sharp conical skimmer samples the centerline of the expansion, which then passes into the differential stage. The optics of the instrument consists of a silicon nitride membrane with a FIB milled pinhole mounted into a metal plate (see inset). The beam is incident on the back of this membrane, leaving a small spot of helium free to strike the sample. The sample itself is able to be rastered underneath the beam via three linear drives to facilitate imaging. A portion of the helium reflected from each point on the sample surface passes through a second aperture in the pinhole plate into the detector chamber, where the stagnation pressure is sampled to yield the intensity.

differential stage, through the internal wall, and encounters the pinhole optics - a focused ion beam (FIB) milled aperture in a silicon nitride membrane (Ted Pella part number 21525). The $250 \times 250$ micron membrane is $200 \mathrm{~nm}$ thick and sits within a $3 \mathrm{~mm}$ silicon disc sealed into an aluminium plate bolted onto the sample chamber side of the internal wall. The sample is kinematically mounted on a stack of three Attocube ECS3030 closed loop piezoelectric slip-stick drives, allowing for 3axis movement in relation to the helium beam. Backscattered helium passes through a $1 \mathrm{~mm}$ diameter aperture in the pinhole plate and travels on to the detector, a Hiden HAL/3F PIC quadrupole with a sensitivity of $1 \times 10^{-4} \mathrm{~A} / \mathrm{mbar}$ for helium (compared to the $3 \times 10^{-5} \mathrm{~A} / \mathrm{mbar}$ sensitivity for the detector used previously ${ }^{12}$ ).

The detector functions in stagnation mode, meaning that based on a constant pump rate the incoming gas will give rise to a stable population which may then be sampled to yield the intensity at that point on the sample surface. ${ }^{10}$ The detector chamber itself is pumped by two Edwards EXT75DX turbo pumps mounted in series, maximising the performance for light gases. A pair of butterfly valves on the detector chamber provide control of the pump rate, allowing for the peak helium signal and the time taken for the pressure to equilibrate to be varied. By rastering the sample back and forth under the beam and measuring the detected helium signal at each point, an image of the sample may be generated.

Of particular note is the care taken in isolating the detector and sample chamber pumping pathways from the high helium throughput source and differential chambers. The undesirable leakage of helium into the detector chamber, possibly even back through the turbomolecular pumps, could degrade the signal-to-background ratio of the instrument. The pumping layout of the new instrument is shown schematically in Figure 2.

\section{RESULTS AND DISCUSSION}

\section{A. Improved count rate}

The most significant improvement to the design over the previous generation of instrument is the reduction in the total length of the beamline. While a longer beamline is traditional in helium atom scatterers to improve the angular resolution

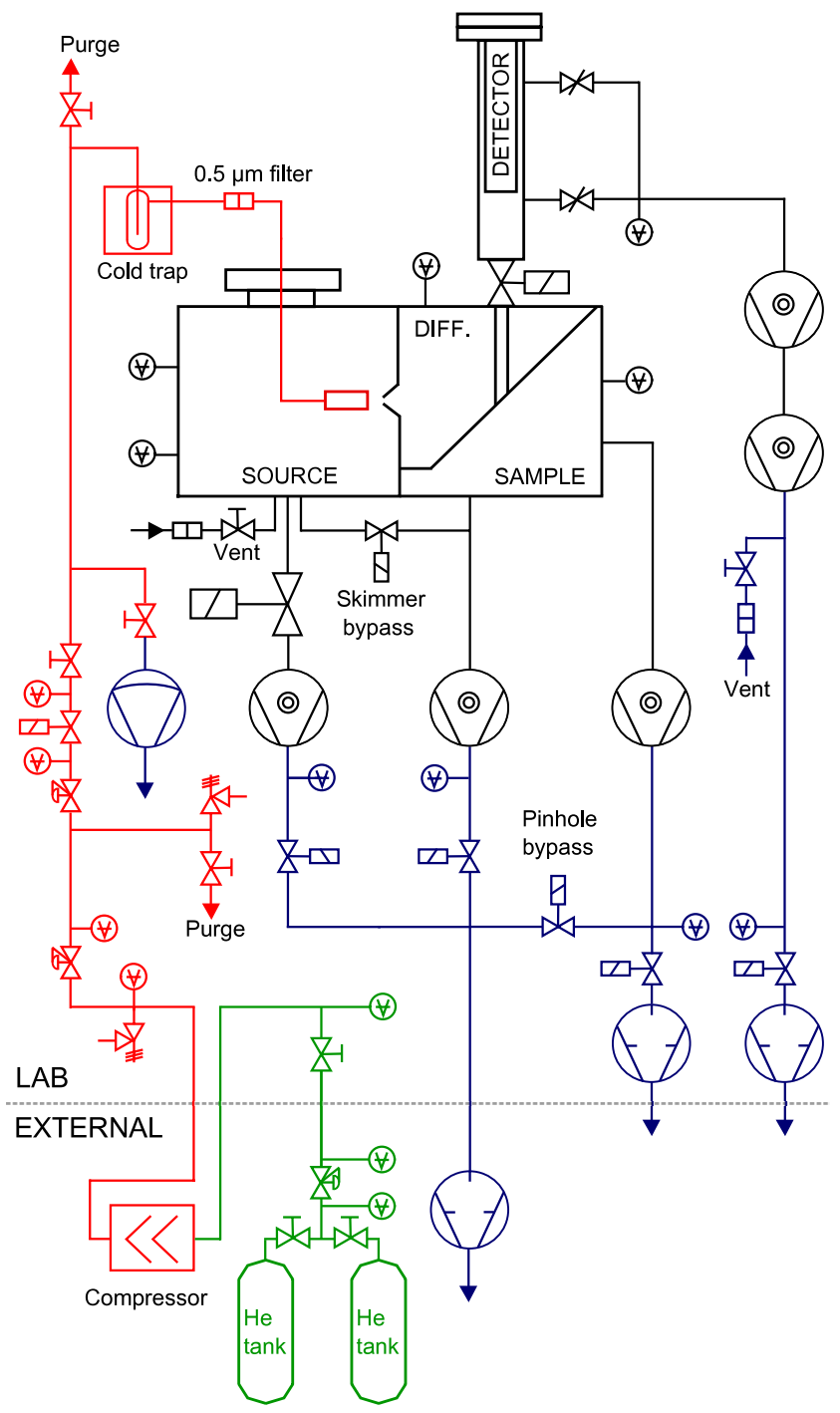

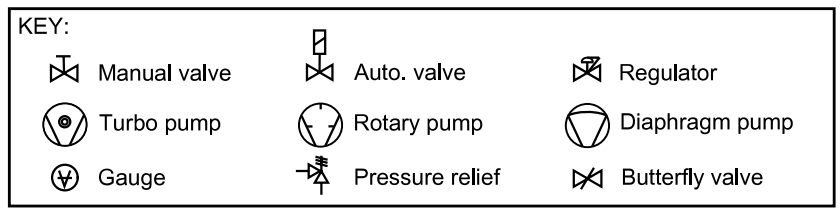

FIG. 2. Simplified gas flow schematic for the SHeM II system. High vacuum stages are shown in black, rough vacuum in blue, high pressure components in red, and gas storage is shown in green. 
and allow for the differential pumping required to lower the background to acceptable levels, ${ }^{13,14}$ it also reduces the intensity reaching the sample. The total SHeM beam path may be subdivided into several smaller sections, namely, the distance from nozzle to skimmer, skimmer to pinhole, pinhole to sample surface (the working distance), and finally from the sample to the detector. Largely due to the change in the shape of the differential pumping chamber, the distance from skimmer to pinhole was able to be considerably reduced while still allowing for a larger effective pump rate. When combined with several other smaller changes, the net result was a reduction in the skimmer to sample distance from $143 \mathrm{~mm}$ to $52 \mathrm{~mm}$.

During the development of the chambers, an analytical model for the instrument using free-molecular gas flow dynamics was employed to test potential geometries. ${ }^{10}$ The model first calculates the centreline intensity of the helium beam as a function of the stagnation conditions including pressure, temperature, and nozzle diameter. The throughput into the differential chamber due to the conductance of the skimmer is then found, with any helium atoms that do not pass through the skimmer becoming part of the background pressure in the source chamber. Based on this background pressure, the leakage rate for helium into the differential stage is also calculated. The process is repeated for the differential stage (with the pinhole forming the orifice to the sample chamber), allowing for the number of helium atoms from the free-jet expansion that go on to strike the sample to be calculated. The reflection of these atoms away from the sample is modelled conservatively through a uniform hemispherical distribution centred about the point where the beam strikes the sample surface. The fraction of this hemispherical cap that is intersected by the detector aperture will then yield the number of helium atoms per unit time that form the signal appropriate for imaging. Table I shows a comparison of the modelled and experimental performance of the new and old SHeM designs.

As shown in Table I, despite the centreline intensity of the beam remaining very similar between design iterations, the count rate for the SHeM II is an order of magnitude larger than the previous generation instrument for the same parameters. Higher count rates allow for the pinhole aperture to be reduced in size (thus improving the resolution of the instrument) while still maintaining practical imaging times. Alternatively, if the pinhole size is kept the same, then the scan time can be reduced appreciably or the counts per pixel raised to produce images with better signal-to-noise ratios. As a demonstration of a typical image from the new instrument, Figure 3 shows sections of a wing from the butterfly species Tirumala hamata as imaged by the SHeM II, along with an optical micrograph for comparison. A butterfly wing is made up of chitinous membranes supported by tubular veins and covered in tiny scales, also formed from chitin. It should be noted that due to the nature of the neutral helium probe, no sample preparation was required (such as the gold coating necessary for such samples in scanning electron microscopy to prevent charging and/or damage). The primary contrast mechanism is topological in nature: the changing mean plane of the wing surface leads to differences in the helium signal directed towards the specular channel, making the ripples through the wing membrane quite obvious. Both shadowing and masking of areas of the sample are evident, in particular by the raised scales along the ridges marking the presence of the veins through the wing. Furthermore, the SHeM image displays excellent depth of field; despite the height of the butterfly wing in Figure 3 varying over almost $2 \mathrm{~mm}$, all the features are in focus, yielding an intuitive image.

\section{B. Effusive beam contribution}

The constricted volume and low pump rate in the differential stage in the SHeM I design meant that an undesirably high helium partial pressure formed behind the pinhole. The effect of this pressure was to generate a secondary effusive beam into the sample chamber with a total intensity of the same order as the supersonic beam. While the nozzle-to-skimmer separation could be adjusted to reduce the intensity of this secondary beam, ${ }^{11}$ it was still a significant contributor to the total helium signal. The secondary beam negatively affects image generation in a number of ways, the foremost being a degradation to the available contrast. Image contrast is dependent on the beam striking a small area of the sample for a given image pixel. The effusive beam spreads out in an isotropic distribution over a sphere (a Lambertian source) and so will form a broad background. For example, with the working distance of the current instrument, the contribution would be almost flat over a typical scanned area (FWHM of $3.6 \mathrm{~mm}$ ). The beam strikes a wide area simultaneously, meaning that the detected signal is a combination of a number of interactions between the probe and sample surface. The broadness of the effusive beam thus constitutes a background helium term without adding to the contrast-producing signal, degrading the image's signal-to-background and signal-to-noise ratios. As such, the secondary beam reduces the effective contrast available between materials, and hence degrades the ability to resolve small or low contrast features. The latter is of particular importance in the effort to investigate the more novel contrast mechanisms such as chemical contrast. ${ }^{15}$ As HAS studies have shown the amount of helium scattered inelastically is 2-3 orders of magnitude lower than that scattered elastically, ${ }^{13,14}$

TABLE I. Modelled vs. typical experimental performance characteristics of the previous (SHeM I) and new (SHeM II) instruments at $200 \mathrm{bar}, 295 \mathrm{~K}$ nozzle stagnation pressure and temperature, respectively.

\begin{tabular}{lcccc}
\hline \hline & SHeM I (model) & SHeM I (expt.) & SHeM II (model) & SHeM II (expt.) \\
\hline Beam centreline & $1.46 \times 10^{20}$ & $(1.4 \pm 0.1) \times 10^{20}$ & $1.45 \times 10^{20}$ & $(1.5 \pm 0.2) \times 10^{20}$ \\
intensity & $\mathrm{He} \mathrm{s}^{-1} \mathrm{sr}^{-1}$ & $\mathrm{He} \mathrm{s}^{-1} \mathrm{sr}^{-1}$ & $\mathrm{He} \mathrm{s}^{-1} \mathrm{sr}^{-1}$ & $\mathrm{He} \mathrm{s}^{-1} \mathrm{sr}^{-1}$ \\
$\begin{array}{l}\text { Detected } \\
\text { count rate }\end{array}$ & $2600 \mathrm{counts} \mathrm{s}^{-1}$ & $1500-4000$ & $34980 \mathrm{counts} \mathrm{s}^{-1}$ & $\begin{array}{c}20000-40000 \\
\text { counts s }\end{array}$ \\
\hline \hline
\end{tabular}



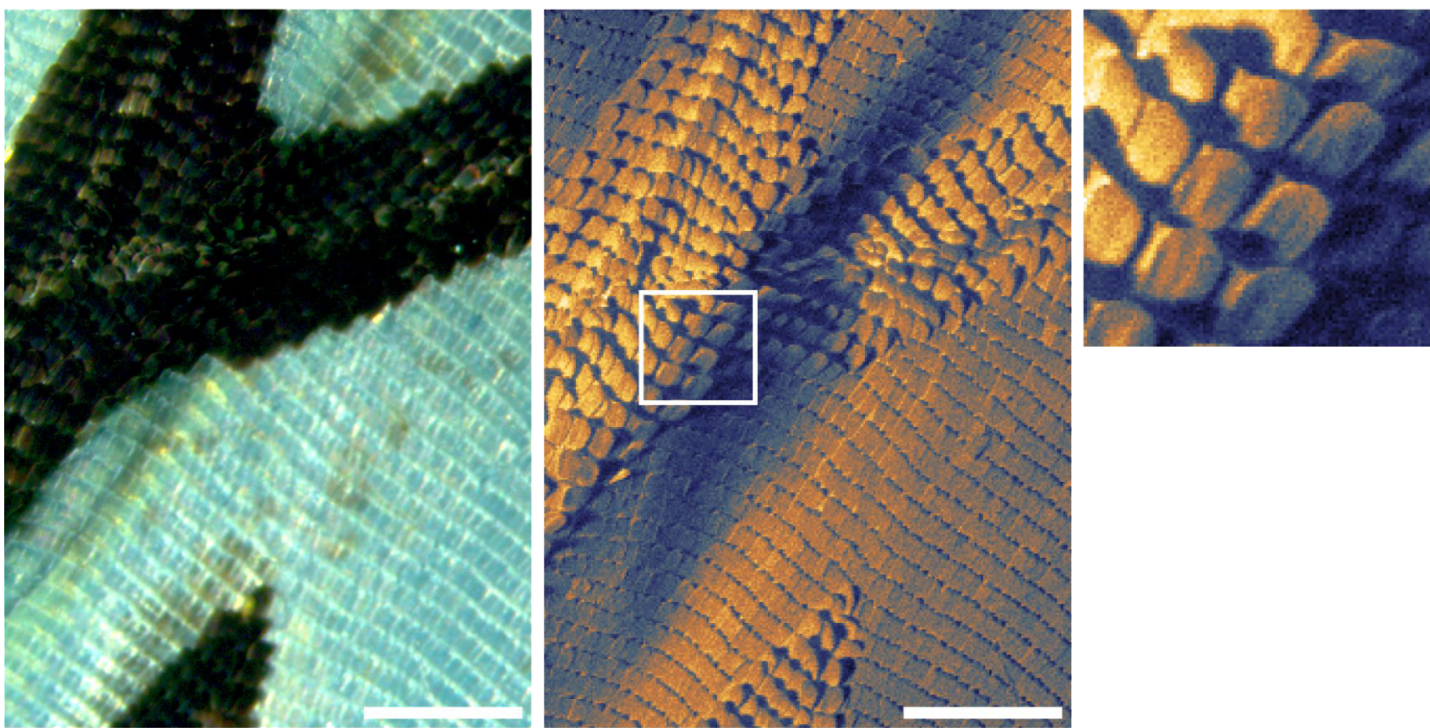

FIG. 3. Matched micrographs of a butterfly's wing (Tirumala hamata). Left: Reflection optical micrograph (Leica M205 C). Center: Scanning helium micrograph as taken by the SHeM II with $8 \mu \mathrm{m}$ steps. Right: Scanning helium micrograph as taken by the SHeM II with $4 \mu \mathrm{m}$ steps; region as indicated by the square in central figure. Both scale bars $600 \mu \mathrm{m}$ in length.

meaning that the contrast mechanisms dependant on such an interaction will be much weaker than topological contrast. An effusive beam contribution will thus render the more delicate contrast mechanisms even more difficult to observe.

With the effects of the secondary beam in mind, efforts were taken to remove the effusive contribution in the new generation instrument. The changes to the differential stage in terms of the design (no longer a tubular construction with a restricted pumping pathway) and the much larger effective pump rate mean that the helium partial pressure in that chamber is much lower during beam operation, reducing the intensity of the effusive beam. Further adjustment of the nozzleto-skimmer position also works to ensure that the supersonic free-jet beam source is the dominant probe. Figure 4 consists of line scans of the nozzle position across the skimmer in the horizontal axis for a range of nozzle to skimmer separations. All data were collected using a cleaned silicon wafer sample set at the instrument working distance $(2.86 \mathrm{~mm})$. It should be noted that the line scan profiles shown in Figure 5 are not explicitly profiles of the helium beam but rather a more complex measurement of the instrument geometry, making direct comparisons to other beam profiles difficult. With the nozzle very close to the skimmer, the differential stage has a higher background helium pressure, causing the secondary beam to become more intense as can be seen in the broad shoulders in the line scan. Increasing the nozzle-to-skimmer distance, even slightly, results in a much sharper peak that is more appropriate for imaging. Comparisons to similar scans for the SHeM I instrument ${ }^{10}$ show a significant reduction in the effusive beam intensity for all nozzle-to-skimmer positions. Campargue ${ }^{16}$ gave that the ideal (i.e., the highest downstream supersonic beam intensity) is achieved at the nozzle-to-skimmer distance $z_{n s}$ given by

$$
Z_{n s}=c d\left(\frac{P_{0} d}{\lambda_{0} P_{b}}\right)
$$

where $d$ is the nozzle diameter, $P_{0}$ is the stagnation pressure, $P_{b}$ is the background source chamber pressure, $\lambda_{0}$ is the stagna- tion mean free path, and $c$ is a number between 0.125 and 0.15 , dependent on the skimmer geometry. ${ }^{17}$ For the SHeM II instrument, the ideal $z_{n s}$ was calculated to be $11.6 \pm 1.1 \mathrm{~mm}$. Based on these calculations and the line scans shown in Figure 5, a nozzle-to-skimmer separation of between 9 and $12 \mathrm{~mm}$ was usual for imaging with the instrument, depending on the level of signal and contrast desired.

In order to demonstrate the effects of the secondary beam on a SHeM micrograph, a section of a curved TEM grid was imaged with and without a strong secondary effusive contribution to the beam profile. The nozzle-to-skimmer distance was initially set to $11 \mathrm{~mm}$ to minimise the effusive beam contri-

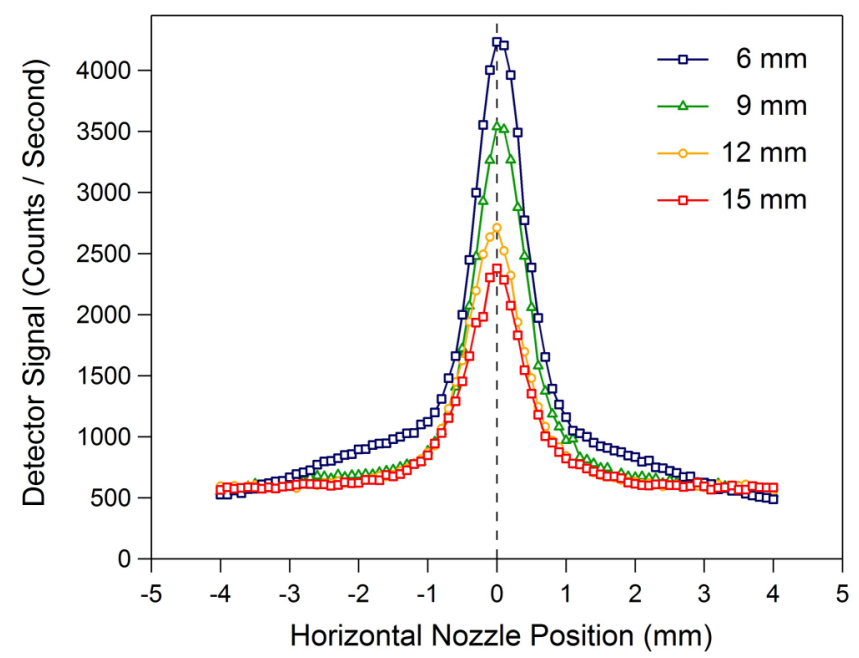

FIG. 4. Line scans of the nozzle in front of the skimmer in the horizontal axis for a series of nozzle-to-skimmer distances. If the nozzle is close to the skimmer $(6 \mathrm{~mm})$, the helium partial pressure in the differential stage increases to the point that a non-trivial secondary beam is produced. This effusive beam can be seen above in the broad shoulders for the $6 \mathrm{~mm}$ line scan, and works to reduce the contrast available from the instrument. Pulling the nozzle back from the skimmer significantly reduces the effusive beam. The nozzle-to-skimmer separations have a zero offset of up to $1 \mathrm{~mm}$ due to the difficulty of aligning the nozzle with the fragile skimmer. 


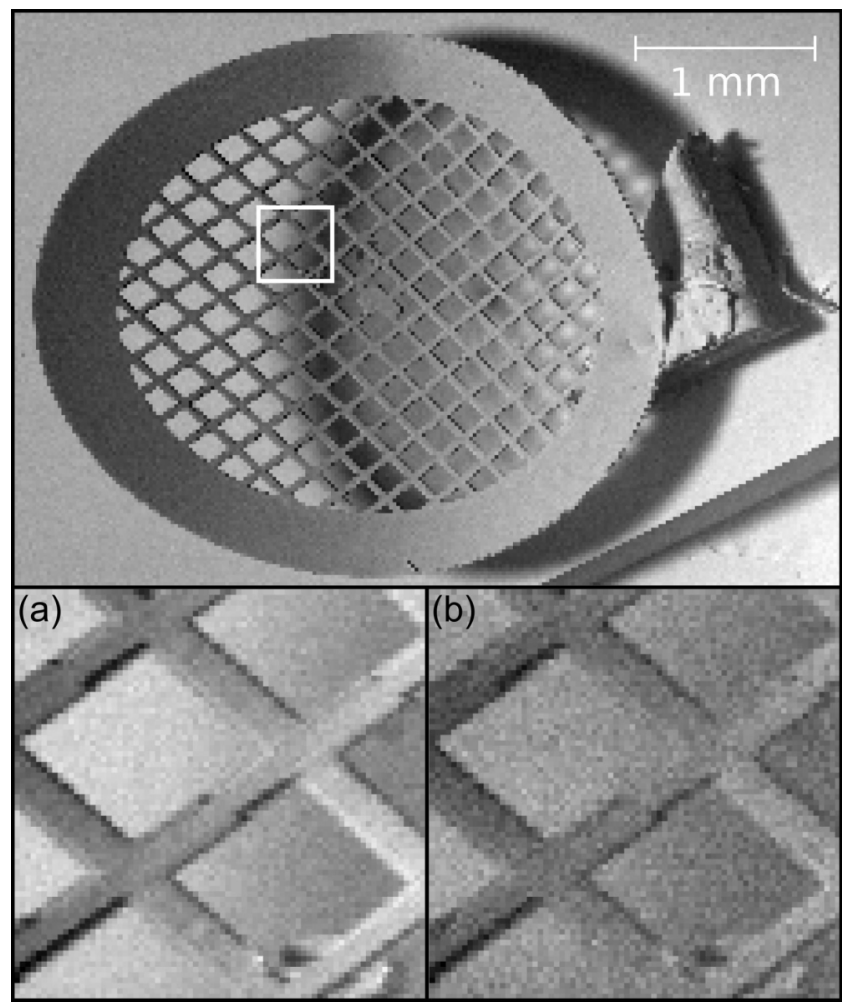

FIG. 5. Top: SHeM micrograph of a TEM grid adhered to a cleaned silicon wafer with a piece of carbon tape. A small section of the grid (as indicated by the white square) was first imaged with a dominant supersonic beam (a), followed by the same supersonic beam along with a significant secondary effusive beam (b). To cause the latter, the differential chamber had additional $4.0 \times 10^{-4}$ millibars of diffuse helium added to give rise to the effusive beam. Note that both (a) and (b) have the same colorbar to allow for a direct comparison. As can be seen, the secondary beam increases the noise in the image and reduces the contrast.

bution in the SHeM II instrument, meaning that the resultant image is due to the sharp supersonic free-jet beam. Next, the intensity of the secondary beam in the previous instrument was replicated by backfilling the differential stage with additional helium gas. To achieve an equivalent pressure behind the pinhole to that of the SHeM I required the introduction of further $4.0 \times 10^{-4}$ millibars of helium into the differential stage (which typically has helium partial pressure of $3.2 \times 10^{-5}$ millibars during beam operation at 200 bar).

Figure 5 shows the images of the grid taken with just the primary beam, and then with the addition of the secondary beam. Table II shows the comparison between the two images in terms of contrast and signal-to-noise. As is evident through direct comparison of the images, both the signal-to-noise and contrast are diminished with the addition of the effusive beam, despite a greater total detected helium count rate. The method used to calculate the signal-to-noise for these images differs slightly from that presented previously with regards to SHeM I. ${ }^{10}$ In this instance, signal-to-noise is calculated by comparing the mean signal to the RMS noise for the same region, namely, the flat silicon substrate. Equivalent analysis on TEM grid images as produced by SHeM I (such as Figure 5(a) in Ref. 10) yields a typical signal-to-noise ratio of $5 \pm 1$. Note that the improvements to the SHeM II design (including the reduced beam path length and higher sensitivity detector) pro-
TABLE II. Comparison of image properties for Figures 5(a) and 5(b).

\begin{tabular}{lcc}
\hline \hline & $\begin{array}{c}\text { Supersonic } \\
\text { beam only }\end{array}$ & $\begin{array}{c}\text { Supersonic with } \\
\text { effusive contribution }\end{array}$ \\
\hline $\begin{array}{l}\text { Michelson contrast } \\
\text { (brightest to darkest) }\end{array}$ & $0.148 \pm 0.008$ & $0.056 \pm 0.004$ \\
\begin{tabular}{l} 
Signal-to-noise \\
\hline
\end{tabular} & $30.62 \pm 8.00$ & $12.12 \pm 2.88$ \\
\hline
\end{tabular}

duce superior signal-to-noise ratios, even with an artificially enhanced secondary beam contribution.

\section{CONCLUSIONS}

A new design of scanning helium microscope has been developed, facilitating an order of magnitude increase in count rates reaching the detector. In addition, the changes to the chambers and pumping considerably reduce the effusive beam contribution which acts to degrade the contrast and signal-tonoise ratio. The design of the SHeM II instrument should allow for the investigation of non-topological contrast modes in the future.

\section{ACKNOWLEDGMENTS}

This research was supported under the Australian Research Council's Discovery Projects (Project No. DP08831308) funding scheme. Postgraduate research scholarships (M.B., A.F.) from the University of Newcastle gratefully acknowledged. We thank the Newcastle and Cavendish workshops and Donald MacLaren for technical support and assistance. This work was performed in part at the Materials node of the Australian National Fabrication Facility, which is a company established under the National Collaborative Research Infrastructure Strategy to provide nano and microfabrication facilities for Australia's researchers.

${ }^{1}$ D. A. MacLaren, B. Holst, D. J. Riley, and W. Allison, Surf. Rev. Lett. 10, 249-255 (2003).

${ }^{2}$ R. B. Doak, R. E. Grisenti, S. Rehbein, G. Schmahl, J. P. Toennies, and C. Wöll, Phys. Rev. Lett. 83, 4229-4232 (1999).

${ }^{3}$ M. M. Greve, A. M. Vial, J. J. Stamnes, and B. Holst, Opt. Express 21, 28483-28495 (2013).

${ }^{4}$ B. Holst and W. Allison, Nature 390, 244 (1997).

${ }^{5}$ P. Sutter, M. Minniti, P. Albrecht, D. Farias, R. Miranda, and E. Sutter, Appl. Phys. Lett. 99, 211907 (2011).

${ }^{6}$ R. B. Doak, J. Phys.: Condens. Matter 16, S2863 (2004).

${ }^{7}$ A. R. Alderwick, A. P. Jardine, H. Hedgeland, D. A. MacLaren, W. Allison, and J. Ellis, Rev. Sci. Instrum. 79, 123301 (2008).

${ }^{8}$ K. M. O'Donnell, A. Fahy, M. Barr, W. Allison, and P. C. Dastoor, Phys. Rev. B 85, 113404 (2012).

${ }^{9}$ P. J. Witham and E. J. Sanchez, J. Microsc. 248, 223 (2012).

${ }^{10}$ M. Barr, A. Fahy, A. Jardine, J. Ellis, D. Ward, D. A. MacLaren, W. Allison, and P. C. Dastoor, Nucl. Instrum. Methods Phys. Res., Sect. B 340, 76-80 (2014).

${ }^{11}$ J. R. Buckland, R. L. Folkerts, R. B. Balsod, and W. Allison, Meas. Sci. Technol. 8, 933-935 (1997).

${ }^{12}$ Hiden Analytical Ltd, private communication (27 July 2012).

${ }^{13}$ Helium Atom Scattering from Surfaces, edited by E. Hulpke (Springer, New York, 1992).

${ }^{14}$ R. B. Doak, in Atomic and Molecular Beam Methods, Volume 2, edited by G. Scoles (Oxford University Press, Oxford, 1992).

${ }^{15}$ D. A. MacLaren and W. Allison, Inst. Phys. Conf. Ser. 179, 383 (2004).

${ }^{16}$ R. Campargue, J. Phys. Chem. 88, 4466-4474 (1984).

${ }^{17}$ H. C. W. Beijerinck, R. J. F. Vangerwen, E. R. T. Kerstel, and J. F. M. Martens, Chem. Phys. 96, 153-173 (1985). 